\title{
Flow-dependent epigenetic DNA methylation regulates endothelial gene expression and atherosclerosis
}

\author{
Jessilyn Dunn, ${ }^{1}$ Haiwei Qiu, ${ }^{1}$ Soyeon Kim, ${ }^{1}$ Daudi Jjingo, ${ }^{2}$ Ryan Hoffman, ${ }^{1}$ \\ Chan Woo Kim, ${ }^{1}$ Inhwan Jang, ${ }^{1}$ Dong Ju Son, ${ }^{1}$ Daniel Kim, ${ }^{1}$ Chenyi Pan, ${ }^{2}$ \\ Yuhong Fan, ${ }^{2}$ I. King Jordan, ${ }^{2}$ and Hanjoong Jo,3
}

\begin{abstract}
${ }^{1}$ Wallace H. Coulter Department of Biomedical Engineering, Georgia Institute of Technology and Emory University, ${ }^{2}$ School of Biology, Georgia Institute of Technology, and ${ }^{3}$ Division of Cardiology, Department of Medicine, Emory University, Atlanta, Georgia, USA.
\end{abstract}

\begin{abstract}
In atherosclerosis, plaques preferentially develop in arterial regions of disturbed blood flow (d-flow), which alters endothelial gene expression and function. Here, we determined that $d$-flow regulates genome-wide DNA methylation patterns in a DNA methyltransferase-dependent (DNMT-dependent) manner. Induction of d-flow by partial carotid ligation surgery in a murine model induced DNMT1 in arterial endothelium. In cultured endothelial cells, DNMT1 was enhanced by oscillatory shear stress (OS), and reduction of DNMT with either the inhibitor 5-aza-2'-deoxycytidine (5Aza) or siRNA markedly reduced OS-induced endothelial inflammation. Moreover, administration of 5 Aza reduced lesion formation in 2 mouse models of atherosclerosis. Using both reduced representation bisulfite sequencing (RRBS) and microarray, we determined that d-flow in the carotid artery resulted in hypermethylation within the promoters of 11 mechanosensitive genes and that $5 \mathrm{Aza}$ treatment restored normal methylation patterns. Of the identified genes, HoxA5 and $\mathrm{Klf} 3$ encode transcription factors that contain cAMP response elements, suggesting that the methylation status of these loci could serve as a mechanosensitive master switch in gene expression. Together, our results demonstrate that d-flow controls epigenomic DNA methylation patterns in a DNMT-dependent manner, which in turn alters endothelial gene expression and induces atherosclerosis.
\end{abstract}

\section{Introduction}

Endothelial cells undergo dramatic gene expression changes when exposed to disturbed blood flow (d-flow) as compared with unidirectional, stable blood flow (s-flow) (1-4). Atherosclerosis preferentially develops in areas of d-flow, where the dysfunctional endothelial cell phenotype initiates and perpetuates plaque development (5-7). S-flow upregulates "atheroprotective" genes and downregulates "proatherogenic" genes, while d-flow enhances proatherogenic genes and suppresses atheroprotective genes. However, the mechanisms by which d-flow causes changes in endothelial cell gene expression are still unclear.

Gene expression can be regulated epigenetically by histone modifications, DNA methylation, and microRNAs (miRNAs) (8). Of these, flow has been shown to regulate gene expression by histone modifications $(9,10)$ and miRNAs $(11-18)$. It is not known, however, whether flow regulates DNA methylation patterns and whether this plays a critical role in mechanosensitive gene expression.

DNA methylation is the most stable epigenetic modification and involves the addition of a methyl group to the $5^{\prime}$ carbon of a cytosine base pair that occurs most often in a CG dinucleotide (CG site) $(20,21)$. CPG islands are dense regions of CG sites that are normally unmethylated and are associated with approximately $40 \%$ of human genes (22-26). However, repetitive elements such as Alu, Line1, and B1 are generally highly methylated

Authorship note: Jessilyn Dunn, Haiwei Qiu, and Soyeon Kim contributed equally to this work.

Conflict of interest: The authors have declared that no conflict of interest exists. Citation for this article: J Clin Invest. 2014;124(7):3187-3199. doi:10.1172/JCI74792.
(24). DNA methylation in the promoter region of a gene, near the transcription start site (TSS), is associated with repression of gene expression (27-29).

DNA methyltransferases (DNMTs) catalyze the addition of the methyl group to cytosine. DNMT1 is classically referred to as a maintenance methylase (it preferentially methylates hemimethylated DNA), although it also has de novo methylation capabilities $(30,31)$. DNMT3a and DNMT3b are referred to as de novo methyltransferases that preferentially add methyl groups to fully unmethylated DNA during development (32). DNA methylation is a gene-regulatory mechanism known to play a key role in various diseases, particularly in cancer, by silencing tumor suppressor genes via aberrant hypermethylation, and drugs that inhibit DNA methyltransferases have proven to be promising treatment options. 5-Aza-2'-deoxycytidine (5Aza, also known as decitabine) is a nucleoside analog that traps DNMT1 in a covalent complex with DNA, and also preferentially targets DNMT1 via ubiquitin-dependent proteasomal degradation, resulting in DNMT1 inhibition (33). 5Aza is an FDA-approved drug and is currently used to treat myelodysplastic syndromes including leukemia (33-38), but its specific mechanism of action and gene targets need to be further determined.

Recently, DNA methylation has been implicated as a novel risk factor for atherosclerosis in smooth muscle cells (39-43). Hypermethylation of the estrogen receptor $\alpha(E R-\alpha)$ promoter, and high plasma homocysteine levels, a source of the methyl group used for DNA methylation, were found in atherosclerosis patients $(44,45)$. 5 -Methylcytosine $(5 \mathrm{mC})$ is elevated in the intima of $A p o E^{-/-}$mice fed a Western diet, and high $5 \mathrm{mC}$ levels are linked to LDL receptor and p53 mutation in vascular cells $(43,46)$. 15-Lipoxygenase, 
A

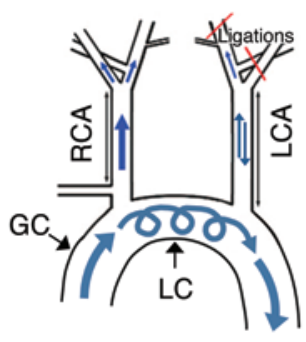

B

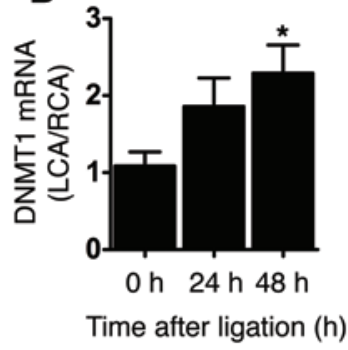

C
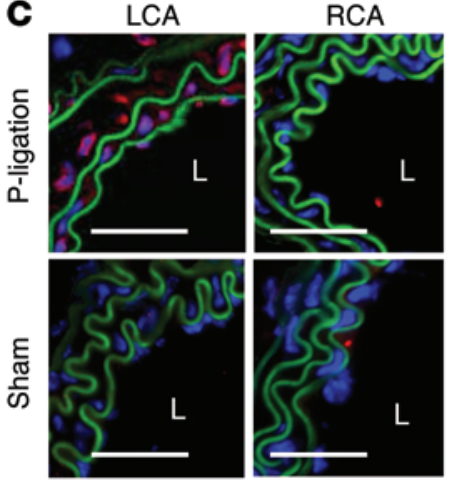

$\mathbf{F}$
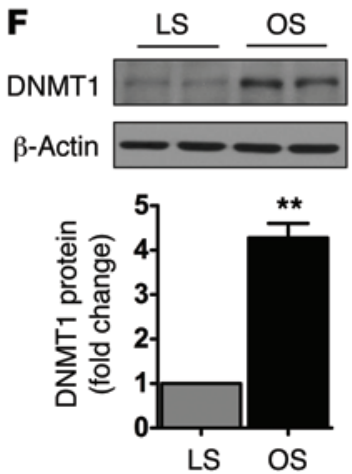

Figure 1

DNMT1 expression is induced by d-flow in endothelial cells in vivo and in vitro. (A) Schematic diagram of the partial carotid ligation model, in which 3 of the 4 caudal branches of the left common carotid artery (LCA) are ligated, while the contralateral right common carotid artery (RCA) remains untouched as an internal control. Also depicted are the naturally, chronically flow-disturbed lesser curvature (LC) and the unidirectionalflow greater curvature (GC). (B) Validation of microarray results by QPCR was performed with endothelial-enriched total RNA obtained from the LCA and RCA at 0, 24, and 48 hours after ligation in C57BL/6 mice. DNMT1 mRNA levels were normalized to 18S. Data are shown as the mean \pm SEM. ${ }^{*} P<0.05, n=8$ (0 hours), $n=9$ (24 hours), $n=12$ (48 hours). (C and D) LCA and RCA frozen sections 48 hours after ligation (scale bars: $50 \mu \mathrm{m})(\mathbf{C})$ and the LC and GC (en face preparation; scale bars: $100 \mu \mathrm{m}$ ) from C57BL/6 mice without ligation surgery (D) were stained with DNMT1 antibody (red). Nuclei are stained with DAPI (blue), and elastic laminae autofluoresce green. The lumen (L) is indicated. (E and F) DNMT1 expression is increased by oscillatory shear stress (OS) compared with laminar shear stress (LS). HUVECs exposed to LS $\left(15 \mathrm{dyn} / \mathrm{cm}^{2}\right)$ or OS $\left( \pm 5 \mathrm{dyn} / \mathrm{cm}^{2}\right.$, at $\left.1 \mathrm{~Hz}\right)$ for 24 hours were used for $\mathrm{PPCR}$ and Western blot analyses for DNMT1 using $18 \mathrm{~S}$ and $\beta$-actin as respective internal controls. The bar graph (F) shows Image J Western blot quantification normalized to $\beta$-actin $(n=7$ each, data are shown as the mean \pm SEM. $\left.{ }^{*} P<0.05 ;{ }^{*} P<0.01\right)$.

a gene implicated in oxidative modification of LDL, is also regulated by DNA methylation (47). It is unknown, however, whether the proatherogenic mechanical force caused by $\mathrm{d}$-flow regulates epigenetic DNA methylation.

Here, we show that DNMT1 is induced by d-flow in endothelial cells both in vivo and in vitro. The DNMT inhibitor 5 Aza prevented endothelial inflammation in vitro and atherosclerosis development in vivo. The genome-scale studies demonstrated that d-flow induces genome-wide DNA methylation pattern changes and revealed novel mechanosensitive genes, such as $\operatorname{Hox} A 5$, that are epigenetically regulated and contain cAMP response elements (CREs).

\section{Results}

D-flow stimulates DNMT1 expression in endothelial cells in vivo and in vitro. We initially identified DNMT1 as a potential mechanosensitive gene in endothelial cells from our previous in vivo mRNA array study (48). To identify flow-sensitive genes in arterial endothelium in vivo, endothelial-enriched RNA was obtained directly from mouse carotid arteries 2 days after partial carotid ligation surgery in C57BL/6 mice (Figure 1A). The mRNA array showed that DNMT1 expression was approximately 2.4 -fold higher in the left carotid artery (LCA), which was partially ligated and exposed to d-flow for 48 hours, than in the contralateral right carotid artery (RCA), exposed to s-flow (48). Here, we validated this endothelial microarray data by quantitative PCR (qPCR), immunostaining, and Western blotting both in vivo and in vitro. DNMT1 mRNA expression showed a significant increase, more than 2-fold, in the LCA endothelium as compared with that of the RCA at 48 hours after partial carotid ligation (Figure 1B). Similarly, robust DNMT1 protein expression was observed in the flow-disturbed LCA (48 hours after ligation) compared with the contralateral RCA and sham-controlled LCA and RCA (Figure 1C). Importantly, a robust DNMT1 protein expression was also observed in endothelial cells in the lesser curvature of the aortic arch, which is naturally and chronically exposed to d-flow without the ligation surgery, but not in the greater curvature, an atherosclerosis-resistant region, which is naturally exposed to s-flow (Figure 1, A and D). Interestingly, DNMT1 expression was increased in the intimal and medial layers of the LCA (Figure 1C), suggesting that d-flow affected the protein expression in both endothelial and smooth muscle cells. These in 
A

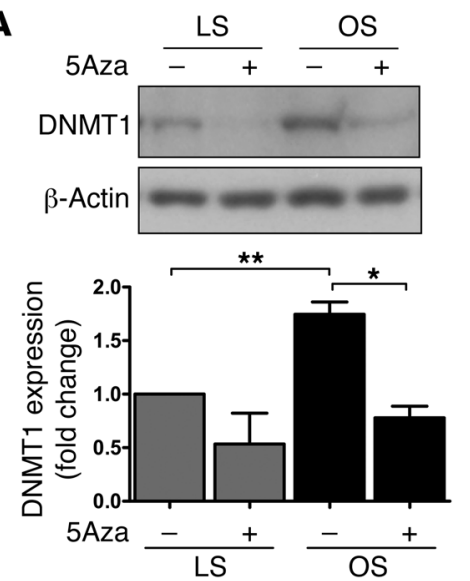

C

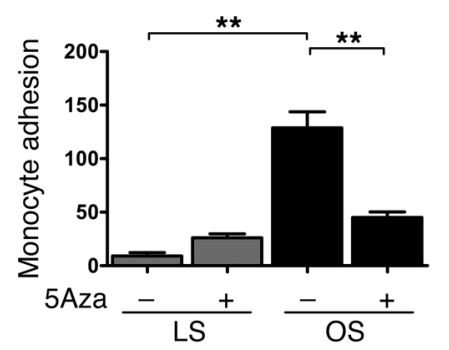

B

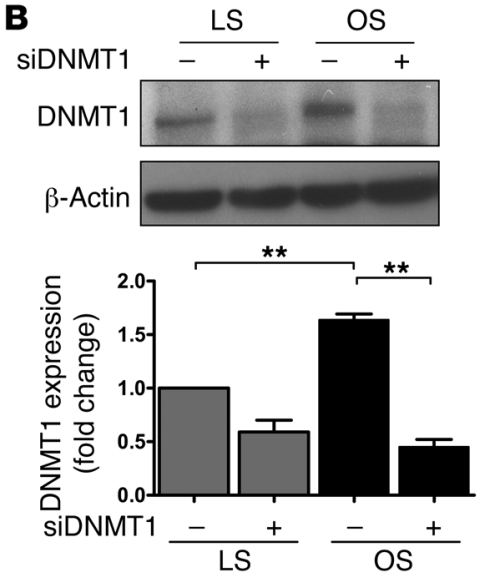

D

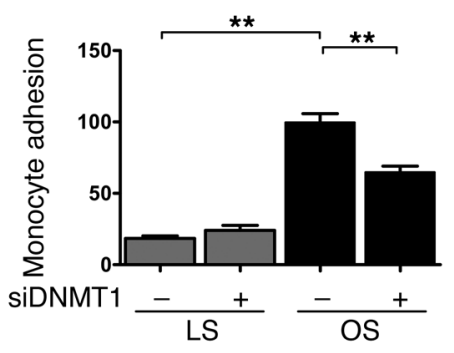

Figure 2

DNMT inhibition blocks OS-induced endothelial inflammation. HUVECs were pretreated with either 5-aza-2'-deoxycytidine (5Aza) at $5 \mu \mathrm{M}$ for 5 days or DNMT1 siRNA (siDNMT1) at $100 \mathrm{nM}$ for 48 hours, and subsequently exposed to OS or LS for an additional 24 hours. (A and B) Cell lysates were then analyzed by Western blotting with the DNMT1 antibody and quantified by Image $J$ analysis using $\beta$-actin as an internal control $\left(n=4\right.$ each, data are shown as the mean \pm SEM. $\left.{ }^{\star} P<0.05 ;{ }^{\star \star} P<0.01\right)$. (C and D) Under the same conditions and following shear, endothelial inflammation was determined by quantification of the number of THP-1 monocytes adhered to sheared endothelial cells ( $n=4$ each, data are shown as the mean SEM. $\left.{ }^{* *} P<0.01\right)$.

vivo data were further confirmed in cultured human endothelial cells (HUVECs) exposed to oscillatory shear stress (OS, mimicking d-flow in vitro) compared with unidirectional laminar shear stress (LS, mimicking s-flow in vitro). DNMT1 mRNA and protein expression was significantly higher in HUVECs exposed to OS as compared with LS (Figure 1, E and F), which directly demonstrates that DNMT1 expression is regulated by OS in endothelial cells. In contrast, expression of the other catalytically active DNMTs, DNMT3a and DNMT3b, was barely detectable and did not show significant changes in protein level in either the partially ligated carotids (LCA vs. RCA) or the naturally flow-disturbed aortic arch (lesser vs. greater curvature) (Supplemental Figure 1; supplemental material available online with this article; doi:10.1172/ JCI74792DS1). These results indicate that the expression of DNMT1, but not DNMT3a or DNMT3b, is regulated in a flowdependent manner in endothelial cells in vivo and in vitro, and therefore subsequent studies focused on the role of DNMT1.

DNMT1 inbibition in endothelial cells by 5 Aza or siRNA inbibits d-flowinduced inflammation in vitro. To determine the functional importance of DNMT1 in endothelial cells, HUVECs were treated with either the DNMT inhibitor 5Aza or DNMT1 siRNA (siDNMT1). As expected, $5 \mathrm{Aza}$ treatment ( $5 \mu \mathrm{M}$ for 5 days) significantly reduced DNMT1, but not DNMT3b, expression (Figure 2A and Supple- mental Figure 2, A and C), and DNMT3a was not detectable by Western blot. Further, 5Aza treatment inhibited DNMT activity in a concentration-dependent manner (Supplemental Figure 2, B and D). As an additional control, we found that $5 \mathrm{Aza}$ treatment significantly blocked global methylation in HUVECs (Supplemental Figure 2E). Exposure to OS increased DNMT1 expression and DNMT activity compared with LS, and 5Aza inhibited this increase (Figure 2A and Supplemental Figure 2, C and D). Treatment with 5 Aza significantly prevented OS-induced monocyte adhesion to HUVECs (Figure 2C). Moreover, siDNMT1 also decreased OS-induced DNMT1 expression as well as monocyte adhesion to HUVECs (Figure 2, B and D). Together, these results suggest that DNMT1 plays a major role in OS-induced endothelial inflammation.

DNMT inhibition by $5 \mathrm{Aza}$ prevents atheroma formation in chronic and acute models of atherosclerosis in mice. We used 2 different $A p o E^{-/-}$mouse models to study the role of DNMT in atherosclerosis: the acute partial carotid ligation model with Western diet, which rapidly develops atherosclerosis within 3 weeks (49), and the conventional Western diet-fed model that chronically develops atherosclerosis in 3 months $(19,50)$. In the carotid ligation model, the LCA rapidly developed atherosclerosis within 3 weeks (Figure 3A), and this was inhibited by $5 \mathrm{Aza}$ treatment in a dose-dependent manner with $0.2 \mathrm{mg} / \mathrm{kg} / \mathrm{d}$ being the lowest effective dose (Figure 3, A and B). Immunostaining for the leukocyte marker CD45 showed a remarkable inhibitory effect of $5 \mathrm{Aza}$ on inflammatory cell infiltration at the $0.2-\mathrm{mg} / \mathrm{kg} / \mathrm{d}$ dose (Supplemental Figure 3A), which was consistent with the atherosclerosis data. In the conventional Western diet model, 5Aza treatment $(0.2 \mathrm{mg} / \mathrm{kg} / \mathrm{d})$ also significantly inhibited atherosclerosis development in the aortic arch and brachiocephalic arterial branch (Figure 3, C and D). In both models, 5Aza treatment did not show a significant effect on the serum lipid profile (Supplemental Figure 4, A and C) and apparent health, but induced a smaller body-weight gain than was seen in the control groups (acute study: $25 \mathrm{~g}$ in control vs. $23 \mathrm{~g}$ in 5 Aza mice at 3 weeks; chronic study: $34 \mathrm{~g}$ in control vs. $31 \mathrm{~g}$ in 5 Aza group at 3 months) (Supplemental Figure 4, B and D). As additional controls, we examined the effect of d-flow, atherosclerosis, and 5Aza treatment on $5 \mathrm{mC}$ content and DNMT1 expression by immunostaining. The dramatically increased $5 \mathrm{mC}$ and DNMT1 levels in the partially ligated LCA at the 3-week time point were significantly reduced by $5 \mathrm{Aza}$ treatment in a dose-dependent manner (Supplemental Figure 3, $\mathrm{B}$ and $\mathrm{C}$ ). Taken together, these results provide strong evidence that 5 Aza treatment prevented atherosclerosis by reducing DNMT expression and activity in the arterial wall.

D-flow induces genome-wide DNA methylation changes and regulates global gene expression in a 5Aza-dependent manner. To determine the mechanisms by which $5 \mathrm{Aza}$ inhibited atherosclerosis in vivo, we tested the hypothesis that d-flow induces hypermethylation of antiatherogenic endothelial cell genes at the promoter regions, thereby silencing their expression, and that 5Aza treatment prevents this hypermethylation, leading to prevention of atherosclerosis. Since it was not known whether d-flow regulates genome-wide DNA meth- 
A
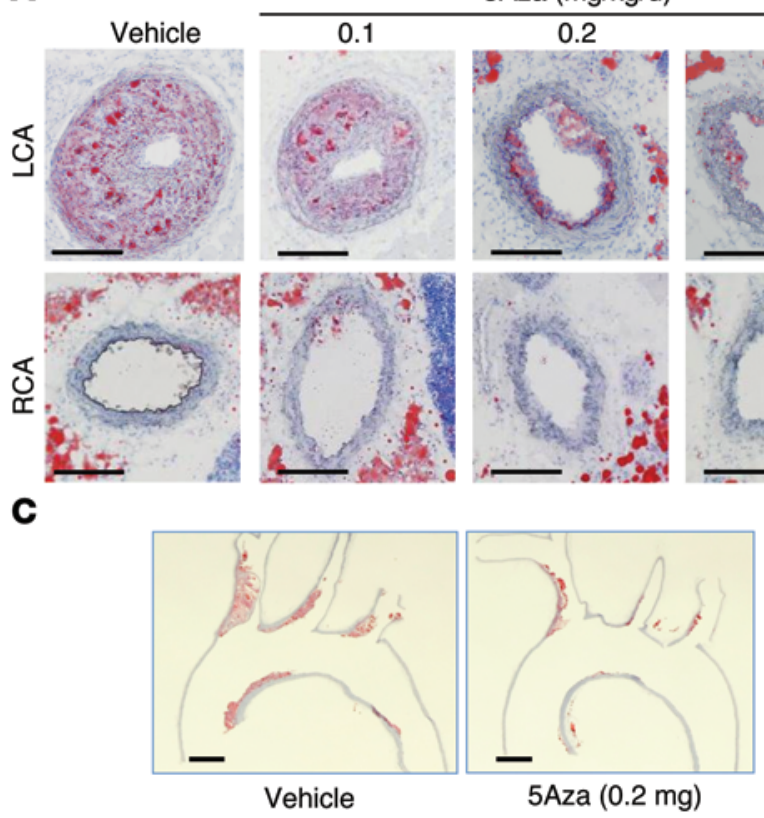

$5 \mathrm{Aza}(\mathrm{mg} / \mathrm{kg} / \mathrm{d})$

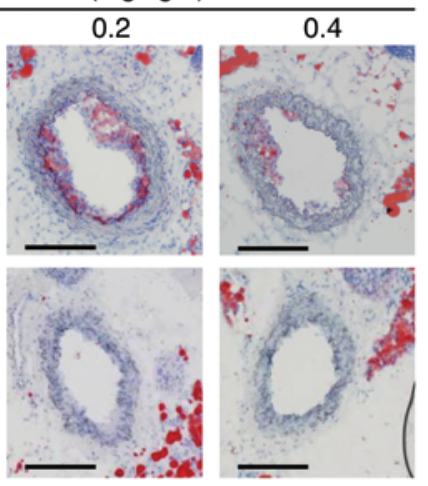

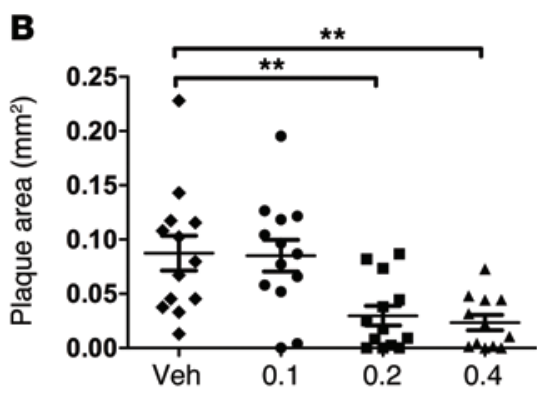

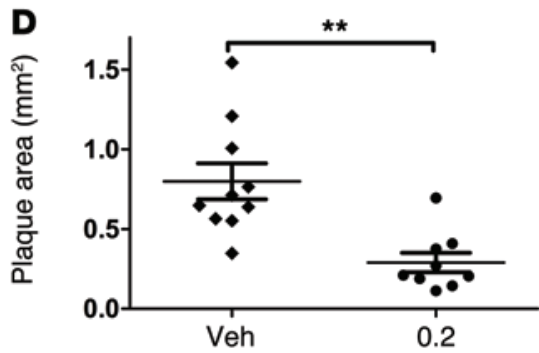

Figure 3

Treatment with the DNMT inhibitor 5Aza inhibits atherosclerosis. (A and B) Acute, partial carotid ligation model of atherosclerosis: $A p o E^{-/-}$mice were given daily i.p. injections of $5 \mathrm{Aza}$ for 1 week at $0.1,0.2$, or $0.4 \mathrm{mg} / \mathrm{kg} / \mathrm{d}$, or saline as a vehicle control. Then, partial carotid ligation was done, and mice were fed a Western diet while continuing the same 5Aza treatment for 3 more weeks. Frozen sections of the carotid arteries were examined by oil red O staining (scale bars: $200 \mu \mathrm{m})(\mathbf{A})$, and plaque area quantification $\left(\mathrm{mm}^{2}\right)$ was done using Image $(\mathbf{B})(n=13$ each, data are shown as the mean \pm SEM. $\left.{ }^{* *} P<0.005\right)$. (C and D) Chronic, diet-induced atherosclerosis model: $A p o E^{-/-}$mice were fed a Western diet (without partial ligation surgery) and treated with $5 \mathrm{Aza}$ (vehicle or $0.2 \mathrm{mg} / \mathrm{kg} / \mathrm{d}$, daily i.p. injections) for 3 months. Aortic arches were longitudinally sectioned and stained with oil red $\mathrm{O}$ (scale bars: $1 \mathrm{~mm})(\mathbf{C})$, and plaque area $\left(\mathrm{mm}^{2}\right)$ was quantified (D) $\left(n=10\right.$ for vehicle, $n=9$ for 5 Aza, mean \pm SEM. $\left.{ }^{\star \star} P<0.005\right)$.

ylation patterns in endothelial cells, we first carried out a reduced representation bisulfite sequencing (RRBS) study using endothelial-enriched genomic DNA (gDNA) obtained from the RCAs and LCAs of partially ligated mice. To further determine whether the flow-dependent DNA methylation changes were regulated in a DNMT-dependent manner, mice treated with 5Aza were compared with a saline vehicle control. Moreover, to determine which mechanosensitive genes were downregulated, potentially by DNA hypermethylation at their promoter, we carried out a concomitant gene transcript microarray study using endothelial-enriched RNA obtained from the LCAs and RCAs of partially ligated mice treated with either saline (RCA vs. LCA) or 5Aza (Aza-RCA vs. Aza-LCA). To rule out the effect of confounding factors such as the hypercholesterolemic condition in $A p o E^{-/-}$mice, C57BL/6 mice were used for these studies. Mice pretreated with $5 \mathrm{Aza}(0.2 \mathrm{mg} / \mathrm{kg} / \mathrm{d})$ or saline for 2 weeks were partially ligated, and the 5 Aza treatment was continued for 1 week after ligation. For the RRBS with next-generation sequencing and gene transcript array studies, gDNA from 20 RCAs versus LCAs and total RNA from 3 RCAs versus LCAs were pooled, respectively. As a control for $5 \mathrm{Aza}$ treatment, blood was collected 1 week after the start of the treatment, and global DNA methylation was measured by high-resolution melt curve (HRM) analysis. This showed that global methylation decreased as early as 1 week after 5Aza treatment, demonstrating the efficacy of this treatment strategy (Supplemental Figure 5, A and B).

The 4 RRBS data sets (RCA, LCA, Aza-RCA, and Aza-LCA) were mapped to the mm9 mouse genome assembly (Build 37, NCBI, July 2007), which contains a total of 21,342,492 CG sites. Our 4 RRBS data sets showed a methylome coverage of approximately $23 \%$ (4,967,716 unique CG sites on average) of all CG sites in the mouse genome, including promoters, gene bodies, and intergenic regions (Supplemental Table 1). Interestingly, approximately 53\% (1,125,997 CG sites on average) of all CG sites in the promoter regions (defined as transcription start site $\pm 1 \mathrm{~kb}$ ) were covered by our RRBS data sets (Supplemental Table 1), which shows a higher coverage in the promoter regions as compared with the other genomic sites.

To measure the DNA methylation changes at each CG site in the RRBS data sets, only those covered in all 4 data sets (RCA, LCA, Aza-RCA, and Aza-LCA) were selected for further analysis (3,232,969 unique CG sites). The methylation ratio at each CG site was compared between the RCA and LCA as well as the Aza-RCA and Aza-LCA using heat-map correlation scatter plots (Figure 4, A and B). Of the 3.2 million CG sites examined, 4.1\% (131,176 CG sites) showed hypermethylation by more than $40 \%$ in the LCA as compared with the RCA, whereas $93 \%$ showed no significant difference in methylation, defined as less than a $40 \%$ difference between the RCA and LCA (Figure 4A). 5Aza treatment reduced the number of hypermethylated CG sites in the LCA by more than $50 \%$ as compared with the saline group (from 131,176 to 55,224 CG sites), while $97 \%$ of the CG sites showed no significant difference between the Aza-RCA and Aza-LCA (Figure 4B). This result showed that the small number (4.1\%) of CG sites that were hypermethylated in the LCA by d-flow was substantially reduced by the 5 Aza treatment (to $1.7 \%$ ), suggesting that d-flow regulates genome-wide DNA methylation patterns in a DNMT-dependent manner. This result was further corroborated by an a priori methylation pattern analysis that 
A

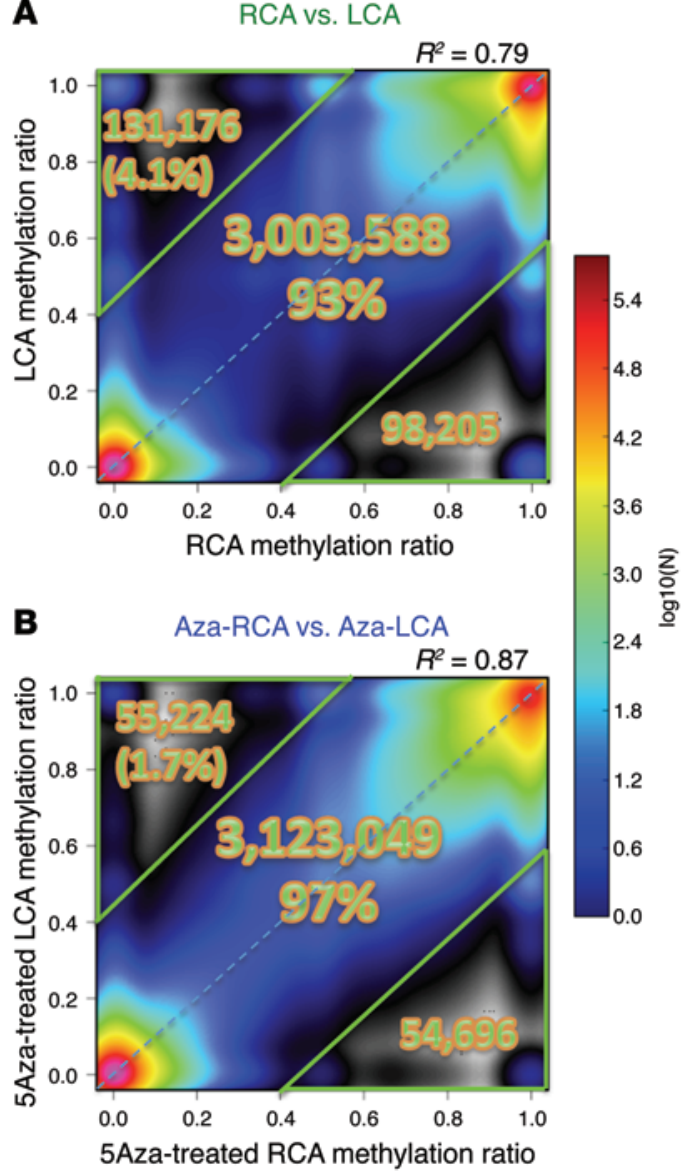

C

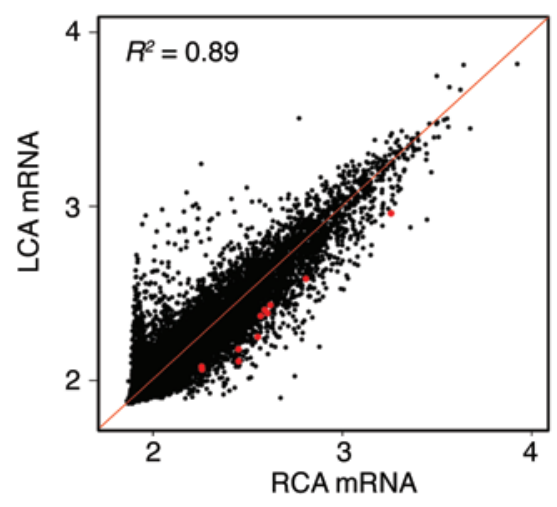

D

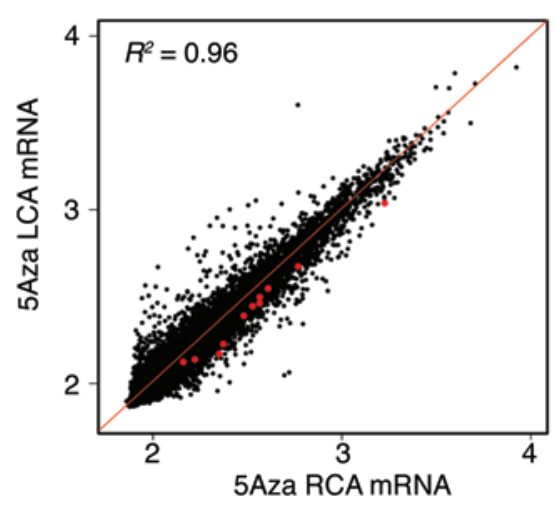

Figure 4

D-flow alters genome-wide DNA methylation patterns and gene expression in a 5Aza-dependent manner. C57BL/6 mice were treated with 5Aza or saline for 2 weeks at $0.2 \mathrm{mg} / \mathrm{kg} / \mathrm{d}$ via i.p. injections. Then, partial carotid ligation was done and mice continued to receive the same 5Aza or saline treatment for 1 more week. (A and B) After sacrifice, endothelial-enriched genomic DNA (gDNA) was collected from the LCA and RCA. gDNA from 20 LCAs and RCAs each was pooled, and the genome-level methylation was analyzed by reduced representation bisulfite sequencing (RRBS). Shown are density heat-map correlation plots portraying the methylation status at each of 3,232,969 CG sites covered by the RRBS analyses. The numbers indicated in the upper, middle, and lower portions of $\mathbf{A}$ indicate $C G$ sites hypermethylated, not altered significantly, and hypomethylated, respectively, in the partially ligated LCA compared with the RCA in saline-treated mice. Likewise, the numbers shown in $\mathbf{B}$ indicate the same as in $\mathbf{A}$, but in the LCAs and RCAs obtained from mice treated with 5Aza. (C and D) After sacrifice, endothelial-enriched RNA obtained from 3 LCAs and RCAs was pooled as 1 sample, and 3 samples per condition (LCA and RCA from saline- vs. 5Aza-treated groups) were analyzed by the Illumina Mouse WG6 microarray. Shown are correlation scatter plots of normalized gene expression detection values on the log scale. The 11 genes identified in Figure 5, A and B, are represented as red dots in each plot.

was performed by ranking the methylation ratio of each gene promoter among the 4 groups. This revealed a pronounced emergent methylation pattern, wherein gene promoters in the LCA $(2,086$ out of 16,493 genes) were hypermethylated as compared with those in the RCA, Aza-LCA, and Aza-RCA (listed in decreasing order of methylation ratios; Supplemental Figure 6A). This supports our hypothesis that d-flow induces promoter hypermethylation in a subset of genes in the LCA in a DNMT-dependent manner.

To determine how many of these mechanosensitive DNA methylation patterns at the promoters correlate to gene expression patterns, we compared the promoter DNA methylation data with the gene transcript array data. First, the gene array data analysis showed that expression of 1,319 genes (569 down- and 750 upregulated) changed by more than $33 \%$ in the LCA compared with the RCA (Figure 4C and Figure 5A). Treatment with 5 Aza decreased the number of differentially expressed genes to 385 (152 down- and 233 upregulated in the LCA) between the Aza-RCA and Aza-LCA (Figure 4D). Importantly, 5Aza treatment rescued the downregulation of 540 of the 569 mechanosensitive genes in the LCA (Figure 4D and Figure 5A), demonstrating the dominant effect of $5 \mathrm{Aza}$ treatment on mechanosensitive gene expression, either directly or indirectly (Figure 4, C and D, Figure 5A, and Supplemental Figure 6, D and E).

Further analysis revealed that our RRBS and gene array data sets showed the expected pattern, wherein percentage promoter methylation inversely correlated with gene expression levels on a global scale (Supplemental Figure $7 \mathrm{~A})$, whereas the same trends were not observed for the gene body and $3^{\prime}$ gene regions surrounding the transcription termination sites (TTSs). This finding is consistent with previous reports showing a close correlation between promoter hypermethylation and gene silencing (51), validating that our RRBS and transcriptome data sets behave in an expected and reliable manner. In addition, as expected, we found that overall promoter CGs were far less methylated $(\sim 29 \%)$ in comparison with genome-wide CGs ( 78\%) (Supplemental Figure 7B). Contrary to our hypothesis, however, the genome-wide and promoter-wide CG methylation status was similar regardless of flow conditions and 5Aza treatment in the RCA, LCA, Aza-RCA, and Aza-LCA (Supplemental Figure $7 \mathrm{~B}$ ), although this result is consistent with the small difference in the number of hypermethylated CG sites on the genome-wide level in LCA and Aza-LCA groups, shown in Figure 4, $\mathrm{A}$ and $\mathrm{B}$. These results suggest a possibility that the $5 \mathrm{Aza}$ effect may be specific and limited to a relatively small number of genes.

Next, we compared the list of mechanosensitive genes that were hypermethylated at their promoter and downregulated in the LCA in a 5 Aza-dependent manner to determine the genes that exhibit 
A

\begin{tabular}{l} 
Promoter DNA methylation \\
\begin{tabular}{|} 
Genes whose promoter is hypermethylated \\
in LCA vs. RCA (421)
\end{tabular} \\
\hline $\begin{array}{c}\text { Genes whose promoter hypermethylation } \\
\text { in LCA was prevented by 5Aza (335) }\end{array}$ \\
\hline
\end{tabular}

11 flow-sensitive genes regulated via promoter methylation
B

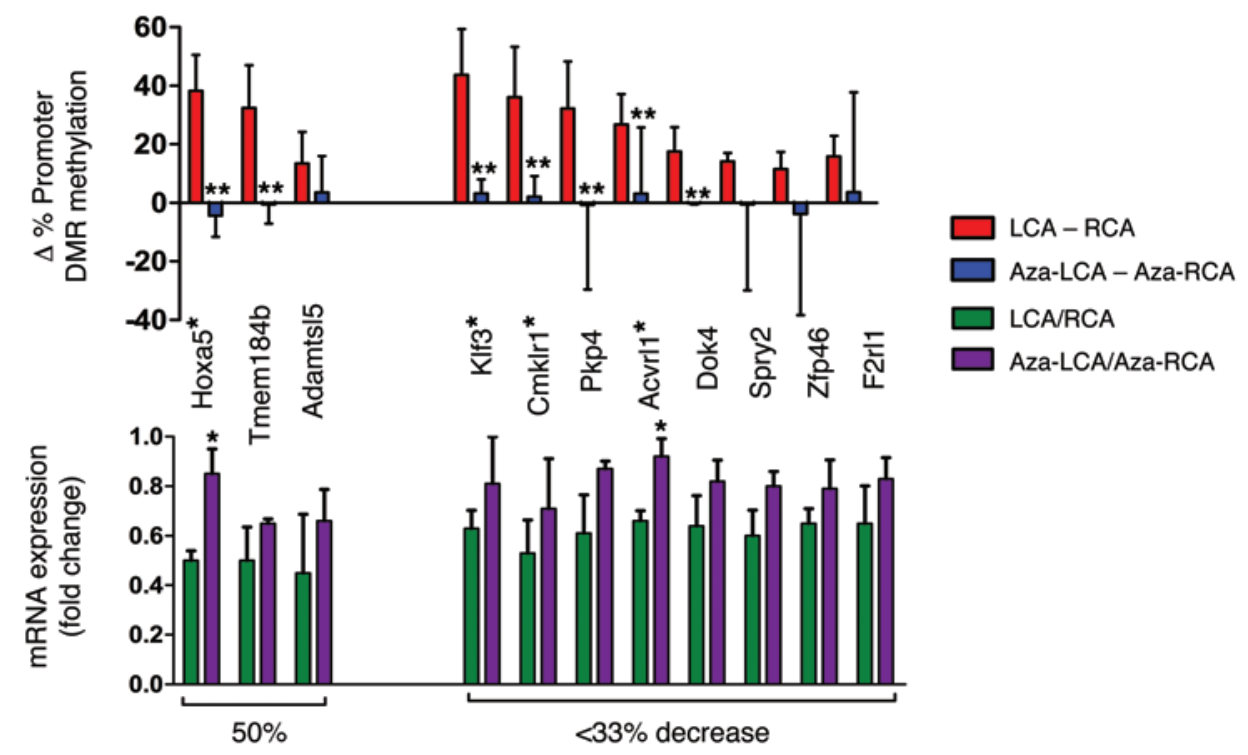

Figure 5

D-flow induces promoter hypermethylation corresponding to suppressed expression in a subset of flow-sensitive genes. (A and B) To identify mechanosensitive genes that are hypermethylated in their promoter and their gene expression downregulated in a $5 \mathrm{Aza}-$ dependent manner, the genes with hypermethylated promoters in the LCA that were rescued by $5 A z a$ treatment were selected. Using the microarray data, the mechanosensitive genes that were downregulated by more than $33 \%$ in the LCA and rescued by $5 A$ za treatment were selected. These 2 selected gene lists were compared with each other, resulting in 11 genes that were hypermethylated in the promoters and downregulated in a 5Aza-dependent manner (data are shown as the mean \pm SEM. ${ }^{*} P<0.05$; $\left.{ }^{* *} P<0.05\right)$. Gene names containing hypermethylated promoter CRE sites in $\mathbf{B}$ are indicated with a single asterisk $\left({ }^{*}\right)$. the expected correlation between methylation and gene expression. This comparative analysis revealed 11 flow-sensitive genes that were hypermethylated at their promoter and silenced by d-flow that could be rescued by 5Aza (Figure 5, A and B, and red points in Figure 4, C and D). These included HoxA5, Tmem184b, Adamtsl5, Klf3, Cmklr1, Pkp4, Acvrl1, Dok4, Spry2, Zfp46, and F2rl1. Notably, HoxA5 showed the most robust flow-dependent changes in promoter methylation and gene silencing that was significantly reversed by $5 \mathrm{Aza}$ treatment, indicating the potential importance of DNMT-dependent mechanosensitive gene regulation by promoter methylation. Detailed analysis of the RRBS DNA methylation data in the HoxA gene cluster is shown in Figure 6A using a University of California, Santa Cruz, genome browser track to display our RRBS data at the nucleotide resolution. The HoxA5 promoter region was clearly hypermethylated in the d-flow LCA as compared with the s-flow RCA, the Aza-LCA, and the Aza-RCA (Figure 6B). This RRBS result was further confirmed by manual bisulfite sequencing of additional endothelial gDNA obtained from the RCA, LCA, Aza-RCA, and Aza-LCA, validating the RRBS data (Figure 7A). Further, we validated the HoxA5 expression data obtained from the microarray study (Figure 7B) by qPCR (Figure 7C). The qPCR result showed that HoxA5 expression was downregulated in the LCA by more than $50 \%$ compared with that in the RCA and was reversed by the 5Aza treatment, validating the microarray result.

Promoter CREs are bypermethylated by d-flow in a 5Aza-dependent manner. To explore whether there are any functional regulatory elements that are common within the promoter regions among the 11 genes, we first searched for transcription factors that are known or predicted to regulate these genes. The systems biological analysis using MetaCore with these 11 genes as the input predicted CREB1 (cAMP response element binding protein) as the transcription factor with the highest potential connectivity. This led us to a computational sequence analysis designed to look for the full palindromic or half CRE sequences (TGACGTCA, TGACG, and CGTCA) within the differentially methylated regions (DMRs) of the 11 gene promoters. This search revealed that 5 of the 11 gene promoter DMRs contained CRE sites, including HoxA5, Klf3, Cmklr1, Acvrl1, and Spry2. We then examined the DNA methylation status of these gene promoter DMRs specifically at the CRE CG site and found that HoxA5, Klf3, Cmklr1, and Acvrl1 indeed showed significant hypermethylation at this CG in the LCA, which was prevented by $5 \mathrm{Aza}$ treatment (Figure 8, A-D). For example, percentage methylation of the HoxA5 promoter CRE CG site was dramatically higher in the LCA (55.6\%) as compared with the RCA (11.5\%), the Aza-LCA (17.2\%), and the Aza-RCA (20.0\%). The promoter CRE CG site in Spry2, however, was not fully covered by our RRBS data; therefore we could not determine its flow- and 5Aza-dependency.

We next examined whether the CREs across the genome display this property of d-flow-induced hypermethylation that can be prevented by $5 \mathrm{Aza}$. We found that CRE sequences across the genome were nearly fully methylated (95\%-100\%) in all 4 groups (Figure 8E). However, CREs within just the promoter regions showed significantly less methylation compared with that of genome-wide CREs (Figure 8F). In the LCA, the average percentage methylation of promoter CREs was $8.7 \%$, which was decreased by approximately $2 \%$ in the Aza-LCA (Figure 8F). In both untreated and 5Aza-treated RCAs, 
Figure 6

RRBS analysis reveals d-flow-induced HoxA5 promoter hypermethylation. (A) DNA methylation data obtained from the RRBS study are shown using a University of California, Santa Cruz, browser track that displays our RRBS data sets. High methylation is denoted in yellow and low methylation in red. (B) A zoomed-in view of the HoxA5 methylation patterns displaying ratios with the number of methylated sequencing reads divided by the total number of sequencing reads at each individual $C G$ site.

promoter CRE methylation was nearly zero, indicating the dramatic effect of s-flow in preventing methylation of promoter CREs in the RCA (Figure 8F). It is interesting to note that $5 \mathrm{Aza}$ treatment showed only a small reduction in average promoter CG site methylation, while it dramatically decreased CG methylation at specific gene promoters such as HoxA5, Klf3, Cmklr1, and Acvrl1, indicating a targeted effect of 5 Aza toward some gene promoters containing CREs.

Given the potential role of the transcription factors HoxA5 and $\mathrm{Klf3}$ as master regulators of gene expression networks, we further examined their relevance to the endothelial flow response. We found that HoxA5 is strongly suppressed in response to d-flow in vivo by microarray and qPCR (Figure 7, B and C, respectively), and to OS in HUVECs in vitro at both the mRNA and the protein level (Figure 9, A and B). Similarly, Klf3 mRNA expression is also suppressed by d-flow in vitro (Figure 9A). We were not able to determine Klf3 protein because of a lack of adequate antibody for Western blot. To analyze the role of these transcription factors in endothelial inflammation, we performed monocyte adhesion assays using siRNAs to HoxA5 and Klf3 in HUVECs under shear. We found that in the LS condition, siRNA-mediated knockdown of HoxA5 significantly increased monocyte adhesion to HUVECs, partially simulating the OS condition of increased inflammation (Figure 9C and Supplemental Figure 8A). However, knockdown of Klf3 did not have a significant effect on endothelial inflammation, suggesting its potential importance in other flow-responsive pathways (Figure 9D and Supplemental Figure 8A).

\section{Discussion}

Here, we demonstrate, for the first time to our knowledge, that blood flow epigenetically controls endothelial gene expression by regulating genome-wide DNA methylation patterns via a DNMT-dependent mechanism. This novel mechanism has broad implications for understanding the atherogenesis mechanisms and identifying potential therapeutic targets of atherosclerosis.

DNMT1 is a mechanosensitive gene upregulated by d-flow, and DNMT inhibition prevents d-flow-induced endothelial inflammation in vitro and atherosclerosis development in vivo. Given the flow-sensitivity of DNMT1, but not DNMT3a or DNMT3b, it is highly likely that DNMT1 plays a dominant role in endothelial inflammation and atherosclerosis. 
A

RCA

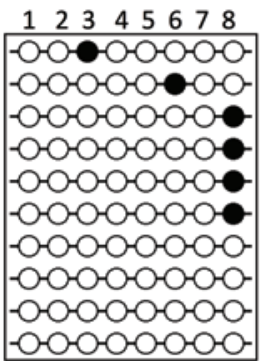

$7.5 \%$

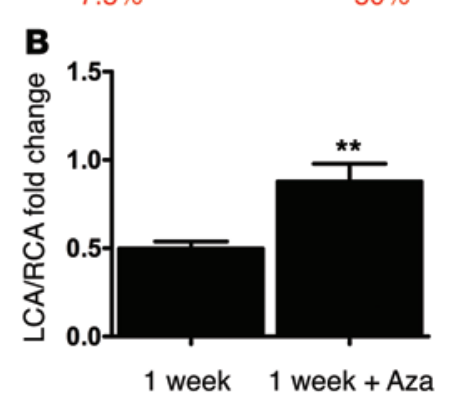

LCA

$50 \%$

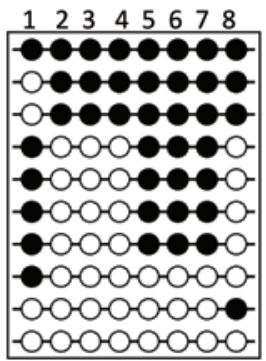

c

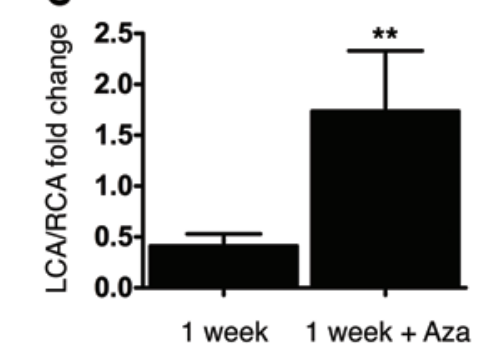

$\mathrm{RCA}+\mathrm{Aza}$

$\mathrm{LCA}+\mathrm{Aza}$

12345678 - -0-0-0-0-0-0- O-O-O-O-O- -0-0-0-0-0-0-0-0-0$17.5 \%$ c $3 \%$
12345678

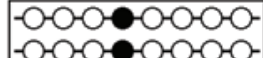

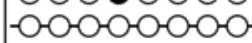
-م-0-0-0- - - - - - - $0-0-0-0-0$

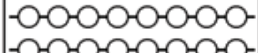

(1)

Figure 7

D-flow induces DNA hypermethylation of the HoxA5 gene promoter and downregulates its expression in a 5 Aza-dependent manner. (A) DNA methylation at the HoxA5 promoter region in endothelial-enriched genomic DNA from the LCA and RCA at 1 week after partial ligation from mice treated with saline or 5 Aza was further examined in independent samples by bisulfite sequencing. Black and white circles represent methylated and unmethylated cytosines, respectively. Eight CG sites (denoted by the columns) were probed in this assay, and 8 to 10 colonies (denoted by the rows) were chosen for analysis. Percentages below the figures denote the average percent methylation for this region of the HoxA5 promoter. (B and C) HoxA5 gene expression was examined in endothelial-enriched RNA from the LCA and RCA obtained at 1 week after partial ligation from saline- or 5Aza-treated mice by microarray analysis. Data for $\mathbf{B}$ are shown as the mean \pm SEM. ${ }^{* \star} P<0.01, n=3$ each and were validated by $\mathrm{qPCR}$. Data for $\mathbf{C}$ are shown as the mean \pm SEM. ${ }^{*} P<0.01, n=5$ each.
We applied a two-pronged parallel approach, using the RRBS and transcriptome studies with in vivo-derived endothelial gDNA and RNA to determine the mechanism by which 5Aza inhibited atherosclerosis. These combined genome-wide studies showed that flow epigenetically regulates gene expression by controlling genomewide DNA methylation patterns in a DNMT-dependent manner. RRBS is widely used in genome-scale methylation studies, although incomplete CG coverage is an inherent limitation of RRBS due to the MspI restriction enzyme digest, PCR, and sequencing steps (52). These limitations produce partial genomic coverage, as evidenced by the $23 \%$ genomic and $53 \%$ promoter coverage of our RRBS assays. Additionally, we were only able to make determinations about genes and genomic regions that were covered in all samples by both the microarray and by RRBS. Therefore, genomic regions with partial coverage between samples or assays were not considered in this particular study, which could have resulted in the overlooking of potentially important CG sites. 5 Aza treatment showed a dramatic effect in rescuing the mechanosensitive gene expression patterns, recovering nearly 540 of 569 genes that were suppressed by d-flow in the LCA (Figure 5A) while having a minimal effect on the RCA gene expression (Supplemental Figure 6E). The lack of any significant effect of 5Aza on the RCA gene expression was surprising, but it may indicate that the $5 \mathrm{Aza}$ effect is limited only to the d-flow-sensitive genes in the LCA. This preferential effect of 5Aza in the LCA may be due to the following reasons: (a) 5Aza in blood is in direct contact with the target cell types, such as arterial endothelium and circulating leukocytes. (b) Arterial regions exposed to d-flow have high endothelial permeability to blood-borne macromolecules, including anti-miRNAs as demonstrated by our group previously (19), albumin (53), and LDL (54). For a similar reason, 5Aza may be preferentially taken up by the endothelial cells in d-flow regions. (c) The endothelial cells in d-flow regions are hyperproliferative, and this facilitates $5 \mathrm{Aza}$ incorporation into these dividing cells' DNA $(55,56)$, while the RCA endothelial cells remain quiescent. This notion is further corroborated by the lack of a widespread effect of $5 \mathrm{Aza}$ on the genome-wide DNA methylation patterns in the RCA, although 5Aza did reduce the number of hypermethylated CG sites significantly for a limited subset of the methylome (from $~ 130,000$ to $\sim 55,000$ sites in the LCA), and those CG sites may have a functional importance in gene expression (Figure 4, A and B).

Using these 2 data sets, our goal was to identify those genes that are hypermethylated in the promoter regions and silenced by $\mathrm{d}$-flow, but that could be rescued by $5 \mathrm{Aza}$ treatment. We would like to emphasize the importance of the user-defined threshold values in our method of gene discovery, which, by using more strict criteria, enabled us to filter out a manageable number of genes for further study. This unbiased genome-wide comparative analysis revealed 11 genes that fit these criteria. This result was surprising at first, since most of the genes downregulated by d-flow in the LCA were rescued by $5 \mathrm{Aza}$ treatment (540 of 569 genes), and because the promoter DNA methylation patterns were rescued by $5 \mathrm{Aza}$ in the majority of genes (335 of 421 genes) (Figure 5A). This discrepancy between the 5 Aza effect on gene expression and methylation and the number of genes that fit our hypothesis could be due to the following possibilities: (a) 5Aza may target a few master regulators such as transcription factors that could in turn regulate the rest of the mechanosensitive genes; (b) there may be only a few key CG sites on promoters that determine the binding of master transcription factors, which control the expression of most of the mechanosensitive genes; and (c) the majority of other CG sites in the promoters do not necessarily lead to functional consequences. To test these possibilities, we first searched for potential master transcription factors that could regulate the 11 mechanosensitive and 5Aza-sensitive genes using the MetaCore analysis. This suggested CREB as a potential master transcription factor that could bind to 5 of the 11 genes. The subsequent computational sequence-based analysis showed that 4 of these genes (HoxA5, Klf3, Cmklr1, and Acvrl1) contained a CG site in the promoter CRE that was hypermethylated by d-flow in a 5Aza-dependent manner (Figure 5B and Figure 8, A-D).

It is interesting that our study finds HoxA5 and Klf3 as novel mechanosensitive transcription factors regulated by DNA methylation that may serve as master regulators of global gene expression 

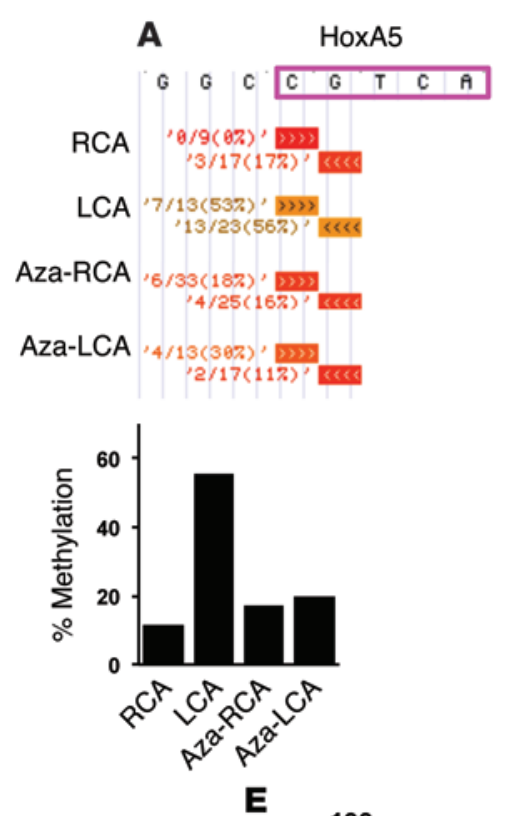

B
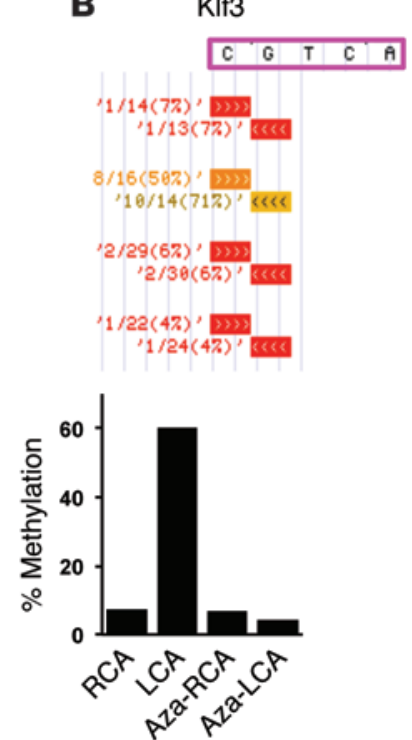

C
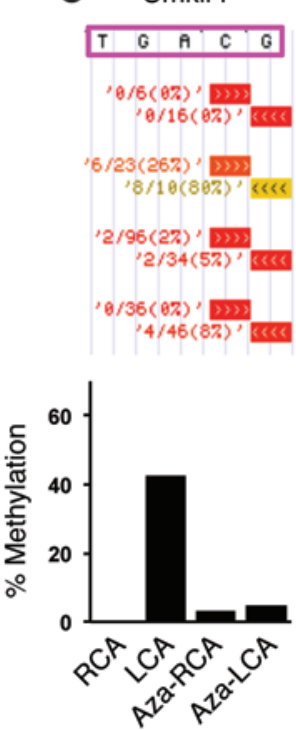

$\mathbf{F}$

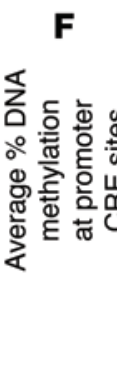

D Acvrl1
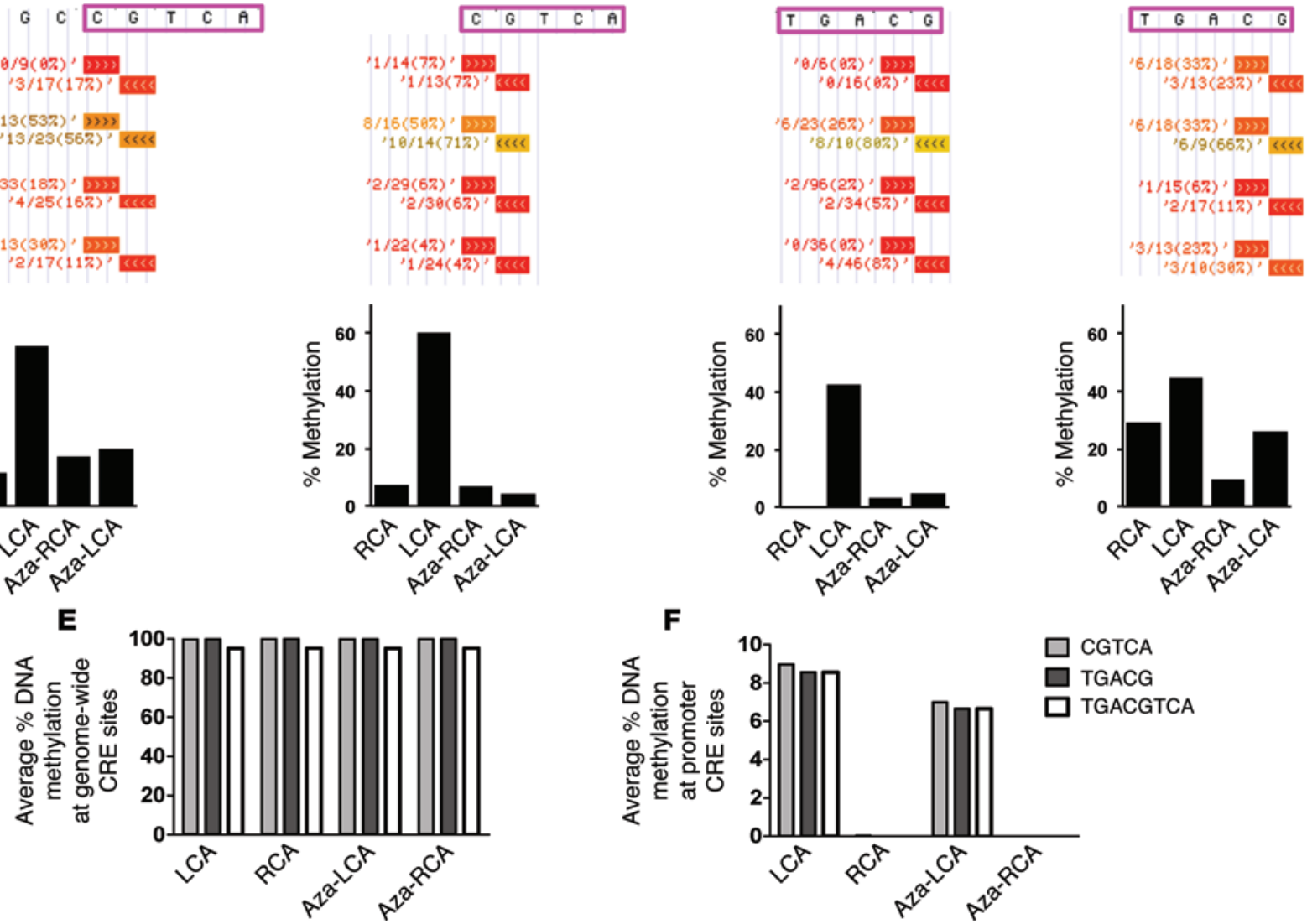

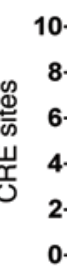

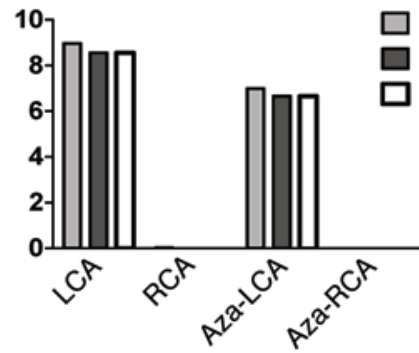

Figure 8

Gene promoters containing CRE are preferentially methylated by d-flow in a 5Aza-dependent manner. (A-D) Of the 11 genes in Figure 5 , HoxA5, KIf3, CmkIr1, and Acvrl1 contain CREs in their promoter differentially methylated regions (DMRs). The methylation ratios (the number of methylated sequencing reads divided by the total number of sequencing reads) for the CG site within the CRE are displayed for each genomic DNA strand for the RCA, LCA, Aza-RCA, and Aza-LCA, and average values are shown below in bar graphs. (E and $\mathbf{F})$ The average methylation status of genome-wide or promoter CREs was calculated as the total number of methylated reads divided by the total number of reads for either genome-wide or promoter CRE CG sites (within $\pm 1 \mathrm{~kb}$ of a gene transcription start site).

in response to flow. Hox genes are highly conserved, are known to be dysregulated in cancer, and are controlled in part by DNA methylation (57-62). Hox family members have been implicated in vascular remodeling, angiogenesis, and disease as orchestrators of gene expression, extracellular matrix, and integrin changes (63). HoxA5 is known to regulate various endothelial functions such as migration, angiogenesis, and inflammation by controlling specific genes (thrombospondin-2, vascular endothelial growth factor receptor-2, ephrin-A1, hypoxia-inducible factor-1 $\alpha$, prostaglandin-endoperoxide synthase-2, and PTEN) (64-68). HoxA5 was shown to regulate expression of approximately 300 genes in breast cancer cell lines (69). Our study shows, for the first time to our knowledge, that HoxA5 is regulated by flow in a DNA methylation-dependent manner and that HoxA5 regulates endothelial inflammation. Interestingly, Klf3 is suppressed in acute myeloid leukemia (AML), and 5Aza is used to treat this disease, although it has not yet been shown whether $5 \mathrm{Aza}$ directly targets Klf3. AML is also treated with all-trans retinoic acid, and, also interestingly, this drug was shown to rescue Klf3 expression by an unknown mechanism (19). Since all-trans retinoic acid is known to influence DNA methylation of a small number of specific genes (70), it would be interesting to test whether Klf3 is regulated by this mechanism in AML. Although our current study focused on examining the role of DNA methylation in vascular biology and disease, these results indicate that our work could have far-reaching implications across multiple diseases.

Our in silico analysis suggests CREB as another key transcription factor whose DNA binding is known to be regulated by methylation of the CRE sites of its target genes, such as HoxA5 and Klf3 $(71,72)$. It was shown previously that protein kinase A downregulation in d-flow leads to decreased CREB phosphorylation and decreased CREB binding to CREs (73). Here, we identified a novel mechanism by which CREB binding to its gene targets may be decreased by d-flow via DNA methylation at their promoter CREs.

One of the most striking findings from our RRBS data is that CRE-localized promoter CG sites were not methylated at all in the s-flow RCA, whereas CG sites across all regions of the promoter were approximately $30 \%$ methylated (Figure 8F and Supplemental Figure $7 \mathrm{~B})$. This suggests that there is an active hypomethylation mechanism that prevents these promoter CRE CG sites from being methylated. Our results also suggest that d-flow induces hypermethylation in these promoter CRE CG sites by a DNMT-dependent mechanism (Figure 8), which in turn has a major impact on gene expression. 
A

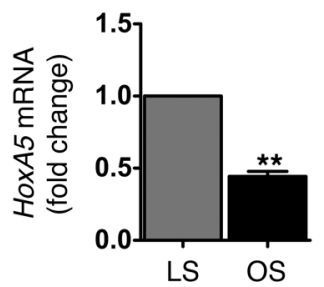

B

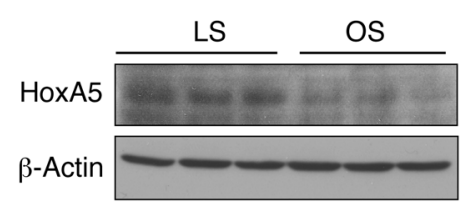

C

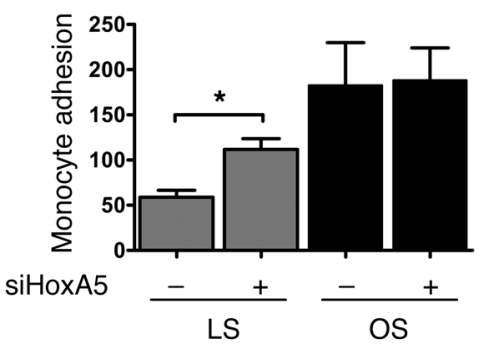

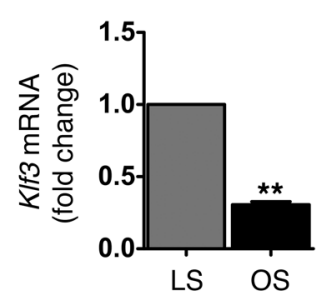
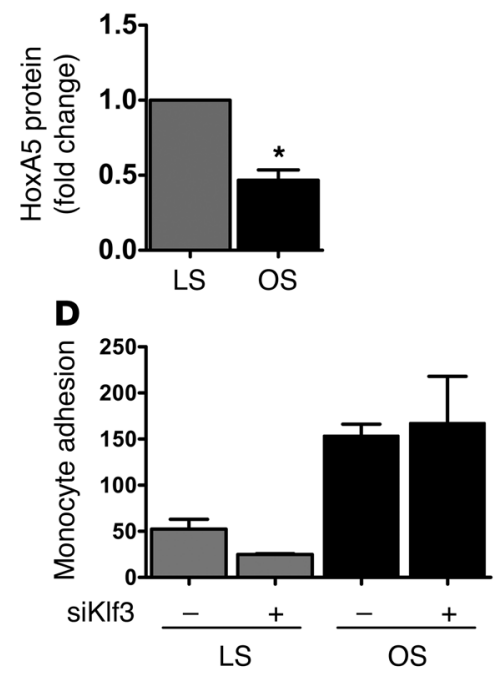

\section{Figure 9}

HoxA5 and KIf3 are suppressed by OS in vitro, and HoxA5 increases LS-suppressed endothelial inflammation. (A) HoxA5 expression and Klf3 expression are suppressed by OS compared with LS. HUVECs exposed to LS $\left(15 \mathrm{dyn} / \mathrm{cm}^{2}\right)$ or OS $\left( \pm 5 \mathrm{dyn} / \mathrm{cm}^{2}\right.$, at $1 \mathrm{~Hz}$ ) for 24 hours were used for qPCR using $18 \mathrm{~S}$ as an internal control $(n=6$ each, data are shown as the mean \pm SEM. ${ }^{\star \star} P<0.01$ ). (B) Western blot analysis for HoxA5 was performed on the same samples from $\mathbf{A}$ using $\beta$-actin as the loading control. The bar graph shows the ImageJ Western blot quantification normalized to $\beta$-actin $(n=3$ each, data are shown as the mean \pm SEM; $\left.{ }^{*} P<0.05\right)$. (C and $\left.\mathbf{D}\right)$ HUVEC were pretreated with siRNA to HoxA5 or Klf3 (100 nM for 24 hours), and subsequently exposed to OS or LS for an additional 24 hours. Following shear, endothelial inflammation was determined by quantification of the number of THP-1 monocytes adhered to sheared endothelial cells $\left(n=3\right.$ each, data are shown as the mean SEM. $\left.{ }^{*} P<0.05\right)$.

In summary, we describe, for the first time to our knowledge, that d-flow-induced changes in global gene expression in endothelial cells are regulated at the epigenomic level in a DNMT- and DNA methylation-dependent manner. We propose that d-flow induces DNMT1 expression in endothelial cells, which in turn stimulates DNA methylation in specific promoter CRE sites of mechanosensitive, master transcription factors including HoxA5 and Klf3. These master genes may then regulate expression of the majority of other mechanosensitive genes, resulting in endothelial dysfunction and atherosclerosis. We further propose that the DNMT inhibitor $5 \mathrm{Aza}$ targets these mechanosensitive master regulators to restore antiatherogenic gene expression profiles. In conclusion, our study provides a novel insight into the mechanism by which flow regulates gene expression in a DNMT-dependent manner and uncovers novel antiatherogenic therapeutic targets.

\section{Methods}

Partial carotid ligation model. Eight-week-old C57BL/6 or ApoE $E^{-/-}$mice (The Jackson Laboratory) were used for all animal studies according to the approved protocol of the IACUC at Emory University. Mice were partially ligated under isoflurane anesthesia and the resultant d-flow conditions in the LCA were determined by ultrasound measurements as described previously (49). Briefly, 3 of 4 caudal branches of the LCA (left external carotid, internal carotid, and occipital artery) were ligated with 6-0 silk suture, while the superior thyroid artery was left intact. Mice were sacrificed by $\mathrm{CO}_{2}$ inhalation and were subsequently perfused with saline containing heparin for 5 minutes. Total carotid intimal RNA or gDNA was isolated by flushing of the RCA and LCA with QIAzol (QIAGEN) or Buffer AL (QIAGEN), respectively, by our method that we have demonstrated to be endothelial-enriched without any significant contamination of smooth muscle cells and leukocytes $(19,48,49)$.

Cell culture and in vitro shear stress system. HUVECs (Lonza cc-2519) were maintained in M199 (Fisher MT10060CV) base culture medium containing 20\% FBS (SH30071), 10\% penicillin/streptomycin/fungizone (Gibco 15240-062), 10\% GlutaMAX (Mediatech 25-005-cl), 10\% endothelial cell growth serum, and $0.4 \%$ heparin (McKesson Medical 404867). In vitro shear stress was applied to passage 6 HUVECs in 10-cm culture plates for 24 hours using a cone and plate viscometer that exerts $15 \mathrm{dyn} / \mathrm{cm}^{2}$ of unidirectional flow (laminar shear stress, or LS, conditions) and $\pm 5 \mathrm{dyn} / \mathrm{cm}^{2}$ of bidirectional flow (oscillatory shear stress, or OS). The cell culture plates were secured in place at the bottom by a vacuum pump, and the incubator was maintained at $5 \% \mathrm{CO}_{2}$ and $37^{\circ} \mathrm{C}$. Total RNA or gDNA was collected by scraping of cells in QIAzol (QIAGEN) or Buffer AL (DNeasy Blood and Tissue Kit; QIAGEN), respectively.

qPCR. Total RNA was collected from HUVEC cultures in $700 \mu \mathrm{l}$ of QIAzol and was purified by the QIAGEN miRNeasy Mini Kit. Total RNA was reverse transcribed into cDNA using SuperScript III and random primers (Invitrogen), as described previously (49). qPCR for specific genes was performed using Brilliant II SYBR Green qPCR Master Mix (Stratagene) with custom-designed primers on the ABI StepOne Plus Real-Time PCR System. Results were normalized to 18S RNA, and the fold change between LCA and RCA was determined using the $2^{\Delta \Delta \mathrm{Ct}}$ method (74). Primer sequences can be found in Supplemental Table 2.

Western blotting. Western blotting was performed by the standard method using antibodies at a 1:1,000 dilution for the following: DNMT1 (sc-20701; Santa Cruz Biotechnology Inc.), DNMT3a (ab23565; Abcam), DNMT3b (ab2851; Abcam), $\beta$-actin (A5316; Sigma-Aldrich), and HoxA5 (ab82645; Abcam).

Tissue preparation, oil red $O$ staining, and immunohistochemistry. Frozen blocks containing the heart, aortic arch, and carotid arteries were prepared in TissueTek and stored at $-80^{\circ} \mathrm{C}$. Sections from the blocks were stained with oil red $\mathrm{O}$ by fixing of the frozen tissues with $10 \%$ formalin for 2 minutes, rinsing twice with distilled water for 5 minutes, orbital shaking in 100\% propanediol for 10 minutes, and incubation in $0.2 \%$ oil red $\mathrm{O}$ solution for 5 minutes at $85^{\circ} \mathrm{C}$. For hematoxylin staining, the previous method was followed by 2 rinses with distilled water, a hematoxylin dip for 30 seconds, 2 rinses with distilled water, 1 rinse with Scott's Tap Water (Sigma-Aldrich), 2 rinses with distilled water, and then mounting. Plaque size was quantified using ImageJ software (NIH). Immunohistochemistry was performed on sections from the frozen blocks using the following antibodies at a 1:50 dilution: 5-methylcytidine (BI-MECY; Eurogentec), DNMT1 (sc-20701; Santa Cruz Biotechnology Inc.), DNMT3b (ab2851; Abcam), DNMT3a (ab23565; Abcam), and CD45 (13-0454; eBioscience). 
$5 A z a$ and siRNA treatment of HUVECs in vitro. Optimal 5-aza-2'-deoxycytidine (5Aza, A3656; Sigma-Aldrich) treatment conditions were determined by a dose curve using $0,0.5,1,5$, and $20 \mu \mathrm{M} 5 \mathrm{Aza}$ for 5 days. DNMT enzymatic activity and DNMT1 protein expression assays indicated the optimal dose of $5 \mu \mathrm{M}$. For shear studies, $5 \mu \mathrm{M} 5 \mathrm{Aza}$ was treated for 5 days before in vitro shear, and the LS or OS conditions were applied for 24 additional hours. siRNA was transfected using Oligofectamine (Invitrogen 12252-011) with either $100 \mathrm{nM}$ siDNMT1, siHoxA5, or siKlf3 (Dharmacon RNAi ON-TARGET plus SMART pool) or $100 \mathrm{nM}$ stealth RNAi negative control (medium GC content, 12935-112; Invitrogen) in OptiMEM (31985-088; Invitrogen) for 6 hours followed by normal HUVEC medium replacement. After 48 hours, shear stress (either LS or OS) was applied for an additional 24 hours.

Quantification of DNMT activity. The DNMT enzymatic activity assay was performed according to the manufacturer's protocol (P-3010; Epigentek).

Restriction enzyme (MspI/HpaII) assay to determine global DNA methylation status in vitro. Genomic DNA was collected and purified by the DNeasy Blood and Tissue kit (QIAGEN) from HUVECs treated with either $5 \mu \mathrm{M}$ $5 \mathrm{Aza}$ or vehicle control for 5 days. Either MspI or HpaII (ER0541; ThermoSci) was reacted with the gDNA according to the recommended protocol for the restriction enzymes. The reaction products were electrophoresed on a $2.5 \%$ agarose gel stained with ethidium bromide alongside a $100-\mathrm{bp}$ ladder (N3231L; New England BioLabs) and imaged under UV light.

Monocyte adhesion assay. HUVECs were pretreated with either 5Aza or siRNA and were subjected to either LS or OS for 24 hours. Separately, human peripheral blood mononuclear leukocytes (THP-1 cells) were cultured in serum-containing RPMI medium (MT10040CM; Fisher) and were labeled in serum-free RPMI with $5 \mu \mathrm{l} / \mathrm{ml}$ 2'7'-bis-(2-carboxyethyl)-5(and-6)-carboxyfluorescein (BCECF-AM, B-1170; Molecular Probes) at $37^{\circ} \mathrm{C}$ for 30 minutes. As a positive control, HUVECs were activated by $1 \mathrm{ng} / \mathrm{ml} \mathrm{TNF-} \alpha$ in serum-free RPMI for 3 hours to induce monocyte adhesion. After shear, each $10-\mathrm{cm}$ plate of HUVECs was exposed to the labeled THP- 1 cells at a concentration of $5 \times 10^{5}$ THP- 1 cells per milliliter (in a total of $6 \mathrm{ml}$ ) for 30 minutes at $37^{\circ} \mathrm{C}$. Nonadherent monocytes were washed away with HBSS (Fisher MT21023CV), and the bound monocytes were fixed with $4 \%$ paraformaldehyde for 5 minutes. Bound monocytes were imaged in 8 fields per plate using fluorescent and bright-field microscopy, and quantification was done by ImageJ software.

$5 A$ za treatment in vivo. Mice were treated by i.p. injection at doses of $0.1,0.2$, or $0.4 \mathrm{mg} / \mathrm{kg} / \mathrm{d}$ of 5 -aza-2'-deoxycytidine (5Aza; Sigma-Aldrich) daily for 4 weeks (the acute d-flow-induced atherosclerosis model), $0.2 \mathrm{mg} / \mathrm{kg} / \mathrm{d}$ for 3 months (the chronic, diet-induced atherosclerosis model), or $0.2 \mathrm{mg} / \mathrm{kg} / \mathrm{d}$ for 3 weeks (the RRBS and array studies). Mouse weight was monitored weekly, and food and water intake and feces were monitored daily.

HRM analysis to quantify global DNA methylation. Global gDNA methylation as represented by genomic repeat element methylation was quantified by LINE1 and B1 high-resolution melt curve (HRM) assays for each mouse in the RRBS and array studies to determine the effect of $5 \mathrm{Aza}$ treatment. HRM analysis was done according to the protocol set by Newman et al. (75). Whole blood was collected by the submandibular technique 1 week after the start of 5 Aza treatment. gDNA was extracted from blood using the DNeasy Blood and Tissue kit (QIAGEN) and was bisulfite converted using the EpiTect Bisulfite Conversion Kit (QIAGEN). Bisulfiteconverted gDNA was subjected to HRM analysis using the F_unmeth_ mLINE1 and R_unmeth_mLINE1, the F_mod_unbiased_B1_Mm, and the R_mod_unbiased_B1_Mm primers from Newman et al. and normal qPCR methods as described above.

RRBS sample collection. Genomic DNA was collected from mouse carotid arteries (as described previously) by flushing with $200 \mu \mathrm{l}$ of a 1:1 solution of Buffer AL and nuclease-free water. gDNA was purified using the DNeasy
Blood and Tissue Kit (QIAGEN), and 20 gDNA samples were pooled per RRBS sample. Pooled gDNA for the RCA, LCA, Aza-RCA, and Aza-LCA were sent to Zymo Research Corp., where reduced representation bisulfite sequencing (RRBS) was carried out by bisulfite conversion, genome reduction by restriction enzyme digest and size selection, library preparation, and next-generation sequencing.

Computational analysis of genome-wide methylome (RRBS) and transcriptome (microarray) data sets. Next-generation sequencing reads from the RRBS study were mapped to the $\mathrm{mm} 9$ genome assembly by Zymo Research Corp. The resulting mapped files were analyzed by our lab. Methylome coverage was determined by division of the number of unique CG sites covered by each individual RRBS data set by the number of total CG sites in the mm9 genome assembly $(21,342,492$ CG sites in chromosomes $1-19, \mathrm{X}$, and Y). Promoter coverage was determined by confining the RRBS and $\mathrm{mm} 9$ assemblies to gene transcription start sites (TSSs, as defined by the NCBI reference sequences database) $\pm 1 \mathrm{~kb}$, and then again calculating coverage in these confined regions.

Heatmap correlation scatter plots were generated using the smoothScatter function in $\mathrm{R}$. The methylation ratios of only those unique CG sites covered by all 4 data sets was plotted, and the $\mathrm{R}^{2}$ values were determined by fitting of linear models with the $\mathrm{R} \operatorname{lm}$ function $(1,76,77)$.

For the a priori emergent methylation pattern analysis, we used only those CG sites confined to promoter regions (TSS $\pm 1 \mathrm{~kb}$ ) and calculated the promoter methylation ratio by the total number of methylated reads divided by the total number of all reads in the region, normalized to the number of CG sites in that region. This was done for each RRBS data set, and then the methylation ratio for each gene promoter was compared between data sets and ranked from highest to lowest methylation ratio. There are 24 possible combinatorial patterns that can result from this ranking analysis by ordering of the 4 samples from highest to lowest, and we counted the number of genes that fell into each of the 24 possible pattern categories.

Gene transcript microarray (transcriptome). Total intimal RNA was collected by flushing of the RCA, LCA, Aza-RCA, and Aza-LCA with $150 \mu$ l of QIAzol and was purified using the miRNeasy Mini Kit (QIAGEN). Three carotid RNA samples were pooled and the combined sample was amplified and reverse transcribed, and the cDNA was applied to Illumina Mouse WG6 microarray chips. The microarray detected 45,281 probes corresponding to 30,854 genes, and the array results are available in the NCBI's Gene Expression Omnibus (GEO) database (GEO GSE56143).

Filtering of RRBS and transcript microarray data sets to discover genes with the hypothesized methylation and expression characteristics. The differentially methylated and differentially expressed genes were parsed according to our hypothesis that d-flow-induced hypermethylation at the promoter suppresses gene expression, and that this could be reversed by $5 \mathrm{Aza}$. We performed thresholding to filter out genes that are hypermethylated in the promoter and suppressed by d-flow in a $5 \mathrm{Aza}$-preventable manner. Specifically, we looked for differentially methylated regions (DMRs) in gene promoters, defined by TSS $\pm 1 \mathrm{~kb}$ containing a minimum of 10 CG sites with a minimum difference in percent methylation $(\Delta \%$ methylation $=\%$ methylation in the LCA - \% methylation in the RCA) of 10 . There were 421 such genes. We further parsed this list to genes whose promoter hypermethylation in LCA was prevented by $5 \mathrm{Aza}(\Delta \%$ methylation $=\%$ methylation in the Aza-LCA - \% methylation in the Aza-RCA < 5), and this resulted in 335 genes. For the mRNA expression criteria, we looked for genes downregulated in LCA versus RCA (LCA/RCA < 0.67), which resulted in 569 genes, and then we further looked for genes whose expression in LCA was rescued by $5 \mathrm{Aza}$ (resulting in 540 genes). By combining these methylation and gene expression criteria, we extracted 11 genes that have d-flow-inducible hypermethylation and gene suppression that can be prevented by $5 \mathrm{Aza}$. 
Of the resulting 11 genes, systems biology analysis via MetaCore (Thomson Reuters) revealed common regulation by the CREB transcription factor in 5 of the genes (only 4 of which had RRBS coverage specifically at the CRE sequence). Subsequently, the RRBS data set was mined to determine the methylation ratio for each sample (RCA, LCA, Aza-RCA, and Aza-LCA) specifically within CRE sequences, and then within those CRE sequences specifically confined to gene promoter regions (TSS $\pm 1 \mathrm{~kb}$ ).

Bisulfite sequencing of HoxA5. HoxA5 bisulfite sequencing primers (Supplemental Table 2) were designed using the MethPrimer program (78). gDNA collected from 4 mouse carotid arteries as described previously was pooled, purified using the DNeasy Blood and Tissue Kit (QIAGEN), and bisulfite converted using the EpiTect Bisulfite Conversion Kit (QIAGEN). Ten nanograms of bisulfite-converted DNA was subjected to PCR amplification using HoxA5 BS-Seq primers at an annealing temperature of $59.5^{\circ} \mathrm{C}$.

Statistics. Statistical analyses were performed using GraphPad Prism 5 (GraphPad Software). Error bars are reported as the SEM. Pairwise comparisons were done using 2-tailed Student's $t$ tests. Multiple comparisons of means were performed using 1-way ANOVA followed by Tukey's multiple comparison tests. Differences between groups were considered significant at $P$ values below $0.05\left(^{*}\right)$, and the $P$ values are denoted separately for values below $0.01(* *)$.

1. Garcia-Cardeña G, Comander J, Anderson KR, Blackman BR, Gimbrone MA Jr. Biomechanical activation of vascular endothelium as a determinant of its functional phenotype. Proc Natl Acad Sci US A. 2001;98(8):4478-4485.

2. McCormick SM, et al. DNA microarray reveals changes in gene expression of shear stressed human umbilical vein endothelial cells. Proc Natl Acad Sci U S A. 2001;98(16):8955-8960.

3. Passerini AG, et al. Coexisting proinflammatory and antioxidative endothelial transcription profiles in a disturbed flow region of the adult porcine aorta. Proc Natl Acad Sci US A. 2004;101(8):2482-2487.

4. Ni CW, et al. Discovery of novel mechanosensitive genes in vivo using mouse carotid artery endothelium exposed to disturbed flow. Blood. 2010; 116(15):e66-e73.

5. Davies PF. Hemodynamic shear stress and the endothelium in cardiovascular pathophysiology. Nat Clin Pract Cardiovasc Med. 2009;6(1):16-26.

6. Chiu JJ, Chien S. Effects of disturbed flow on vascular endothelium: pathophysiological basis and clinical perspectives. Physiol Rev. 2011;91(1):327-387.

7. Tarbell JM, Shi Z-D, Dunn J, Jo H. Fluid mechanics, arterial disease, and gene expression. Annu Rev Fluid Mech. 2014;46:591-614.

8. Robertson KD. DNA methylation and chromatin - unraveling the tangled web. Oncogene. 2002; 21(35):5361-5379.

9. Illi B, et al. Shear stress-mediated chromatin remodeling provides molecular basis for flow-dependent regulation of gene expression. Circ Res. 2003; 93(2):155-161.

10. Illi B, et al. Epigenetic histone modification and cardiovascular lineage programming in mouse embryonic stem cells exposed to laminar shear stress. Circ Res. 2005;96(5):501-508.

11. Ni CW, Qiu H, Jo H. MicrorRNA-663 upregulated by oscillatory shear stress plays a role in inflammatory response of endothelial cells. Am J Physiol Heart Circ Physiol. 2011;300(5):H1762-H1769.

12. Qin X, et al. MicroRNA-19a mediates the suppressive effect of laminar flow on cyclin D1 expression in human umbilical vein endothelial cells. Proc Natl Acad Sci U S A. 2010;107(7):3240-3244.

13. Weber M, Baker MB, Moore JP, Searles CD. MiR-21 is induced in endothelial cells by shear stress and modulates apoptosis and eNOS activity. Biochem Biophys Res Commun. 2010;393(4):643-648.

14. Fang Y, Shi C, Manduchi E, Civelek M, Davies PF.
MicroRNA-10a regulation of proinflammatory phenotype in athero-susceptible endothelium in vivo and in vitro. Proc Natl Acad Sci U S A. 2010; 107(30):13450-13455.

15. Marin T, et al. Mechanosensitive microRNAs-role in endothelial responses to shear stress and redox state. Free Radic Biol Med. 2013;64:61-68.

16 . Wu W, et al. Flow-dependent regulation of Krüppel-like factor 2 is mediated by microRNA-92a. Circulation. 2011;124(5):633-641.

17. Wei Y, Nazari-Jahantigh M, Neth P, Weber C, Schober A. MicroRNA-126,-145, and -155: a therapeutic triad in atherosclerosis? Arterioscler Thromb Vasc Biol. 2013;33(3):449-454.

18. Son DJ, et al. The atypical mechanosensitive microRNA-712 derived from pre-ribosomal RNA induces endothelial inflammation and atherosclerosis. Nat Commun. 2013;4:3000.

19. Humbert M, et al. Deregulated expression of Kruppel-like factors in acute myeloid leukemia. Lenk Res. 2011;35(7):909-913.

20. Jeltsch A. Beyond Watson and Crick: DNA methylation and molecular enzymology of DNA methyltransferases. Chembiochem. 2002;3(4):274-293.

21. Eckhardt F, et al. DNA methylation profiling of human chromosomes 6, 20 and 22. Nat Genet. 2006; 38(12):1378-1385.

22. Gardiner-Garden M, Frommer M. CPG islands in vertebrate genomes. J Mol Biol. 1987;196(2):261-282.

23. Bestor TH, Gundersen G, Kolstø AB, Prydz H. CpG islands in mammalian gene promoters are inherently resistant to de novo methylation. Genet Anal Tech Appl. 1992;9(2):48-53.

24. Takai D, Jones PA. Comprehensive analysis of CPG islands in human chromosomes 21 and 22. Proc Natl Acad Sci U S A. 2002;99(6):3740-3745.

25. Strichman-Almashanu LZ, et al. A genome-wide screen for normally methylated human CpG islands that can identify novel imprinted genes. Genome Res. 2002;12(4):543-554.

26. Rollins RA, et al. Large-scale structure of genomic methylation patterns. Genome Res. 2006; 16(2):157-163.

27. Song F, et al. Association of tissue-specific differentially methylated regions (TDMs) with differential gene expression. Proc Natl Acad Sci U S A. 2005; 102(9):3336-3341.

28. Grunau C, Hindermann W, Rosenthal A. Largescale methylation analysis of human genomic DNA reveals tissue-specific differences between the methylation profiles of genes and pseudogenes. Hum Mol Genet. 2000;9(18):2651-2663.

29. Weber M, et al. Chromosome-wide and promoter-specific analyses identify sites of differential DNA methylation in normal and transformed human cells. Nat Genet. 2005;37(8):853-862.

30. Bestor TH. The DNA methyltransferases of mammals. Hum Mol Genet. 2000;9(16):2395-2402.

31. Hsieh CL. The de novo methylation activity of Dnmt3a is distinctly different than that of Dnmt. BMC Biochem. 2005;6:6.

32. Okano M, Li E. Genetic analyses of DNA methyltransferase genes in mouse model system. J Nutr. 2002;132(8 suppl):2462S-2465S.

33. Ghoshal K, et al. 5-Aza-deoxycytidine induces selective degradation of DNA methyltransferase 1 by a proteasomal pathway that requires the KEN box, bromo-adjacent homology domain, and nuclear localization signal. Mol Cell Biol. 2005;25(11):4727-4741.

34. Mossman D, Kim KT, Scott RJ. Demethylation by 5 -aza-2'-deoxycytidine in colorectal cancer cells targets genomic DNA whilst promoter $\mathrm{CPG}$ island methylation persists. BMC Cancer. 2010;10:366.

35. Liu Z, et al. 5-Aza-2'-deoxycytidine induces retinoic acid receptor- $\beta 2$ demethylation and growth inhibition in esophageal squamous carcinoma cells. Cancer Lett. 2005;230(2):271-283.

36. Christman JK. 5-Azacytidine and 5-aza-2'-deoxycytidine as inhibitors of DNA methylation: mechanistic studies and their implications for cancer therapy. Oncogene. 2002;21(35):5483-5495.

37. Issa JP, et al. Phase II study of low-dose decitabine in patients with chronic myelogenous leukemia resistant to imatinib mesylate. J Clin Oncol. 2005; 23(17):3948-3956.

38. Lemaire $M$, et al. Importance of dose-schedule of 5 -aza-2'-deoxycytidine for epigenetic therapy of cancer. BMC Cancer. 2008;8:128.

39. Turunen MP, Ylä-Herttuala S. Epigenetic regulation of key vascular genes and growth factors. Cardiovasc Res. 2011;90(3):441-446.

40. Nazarenko MS, Puzyrev VP, Lebedev IN, Frolov AV, Barbarash OL, Barbarash LS. Methylation profiling of DNA in the area of atherosclerotic plaque in humans. Mol Biol. 2011;45(4):561-566.

41. Wierda RJ, Geutskens SB, Jukema JW, Quax $\mathrm{PH}$, van den Elsen PJ. Epigenetics in atherosclerosis and inflammation. J Cell Mol Med. 2010; 14(6A):1225-1240.

42. Hiltunen MO1, Ylä-Herttuala S. DNA methylation, 
smooth muscle cells, and atherogenesis. Arterioscler Thromb Vasc Biol. 2003;23(10):1750-1753.

43. Hiltunen MO, et al. DNA hypomethylation and methyltransferase expression in atherosclerotic lesions. Vasc Med. 2002;7(1):5-11.

44. Jamaluddin MS, Yang X, Wang H. Hyperhomocysteinemia, DNA methylation and vascular disease. Clin Chem Lab Med. 2007;45(12):1660-1666.

45. Huang YS, Zhi YF, Wang SR. Hypermethylation of estrogen receptor-alpha gene in atheromatosis patients and its correlation with homocysteine. Pathophysiology. 2009;16(4):259-265.

46. Rideout WM 3rd, Coetzee GA, Olumi AF, Jones PA. 5-Methylcytosine as an endogenous mutagen in the human LDL receptor and p53 genes. Science. 1990; 249(4974):1288-1290.

47. Liu C, Xu D, Sjöberg J, Forsell P, Björkholm M, Claesson HE. Transcriptional regulation of 15-lipoxygenase expression by promoter methylation. Exp Cell Res. 2004;297(1):61-67.

48. Ni CW, et al. Discovery of novel mechanosensitive genes in vivo using mouse carotid artery endothelium exposed to disturbed flow. Blood. 2010; 116(15):e66-e73.

49. Nam D, et al. Partial carotid ligation is a model of acutely induced disturbed flow, leading to rapid endothelial dysfunction and atherosclerosis. Am J Physiol Heart Circ Physiol. 2009;297(4):H1535-H1543.

50. Park JG, et al. Peroxiredoxin 2 deficiency exacerbates atherosclerosis in apolipoprotein E-deficient mice. Circ Res. 2011;109(7):739-749.

51. Jjingo D, Conley AB, Yi SV, Lunyak VV, Jordan IK. On the presence and role of human gene-body DNA methylation. Oncotarget. 2012;3(4):462-474.

52. Gu H, et al. Genome-scale dna methylation mapping of clinical samples at single-nucleotide resolution. Nat Methods. 2010;7(2):133-136.

53. Chien S, Lin SJ, Weinbaum S, Lee MM, Jan KM. The role of arterial endothelial cell mitosis in macromolecular permeability. Adv Exp Med Biol. 1988; 242:59-73.

54. Herrmann RA, Malinauskas RA, Truskey GA. Characterization of sites with elevated LDL permeability at intercostal, celiac, and iliac branches of the normal rabbit aorta. Arterioscler Thromb. 1994; 14(2):313-323

55. Foteinos G, Hu Y, Xiao Q, Metzler B, Xu Q. Rapid endothelial turnover in atherosclerosis-prone areas coincides with stem cell repair in apolipoprotein E-deficient mice. Circulation. 2008;117(14):1856-1863.

56. Schwartz SM, Benditt EP. Aortic endothelial cell replication. I. Effects of age and hypertension in the rat. Circ Res. 1977;41(2):248-255.

57. Tanzer A, Amemiya CT, Kim CB, Stadler PF. Evolution of microRNAs located within Hox gene clusters. J Exp Zool B Mol Dev Evol. 2005;304(1):75-85.

58. Illingworth $\mathrm{R}$, et al. A novel $\mathrm{CPG}$ island set identifies tissue-specific methylation at developmental gene loci. PLoS Biol. 2008;6(1):e22.

59. Rossig L, et al. Histone deacetylase activity is essential for the expression of HoxA9 and for endothelial commitment of progenitor cells. J Exp Med. 2005;201(11):1825-1835.

60. Han L, Witmer PD, Casey E, Valle D, Sukumar S. DNA methylation regulates MicroRNA expression. Cancer Biol Ther. 2007;6(8):1284-1288.

61. Pei L, et al. Genome-wide DNA methylation analysis reveals novel epigenetic changes in chronic lymphocytic leukemia. Epigenetics. 2012;7(6):567-578.

62. Rauch TA, Wu X, Zhong X, Riggs AD, Pfeifer GP. A human B cell methylome at 100-base pair resolution. Proc Natl Acad Sci U S A. 2009;106(3):671-678.

63. Gorski DH, Walsh K. The role of homeobox genes in vascular remodeling and angiogenesis. Circ Res. 2000;87(10):865-872.

64. Lee JY, et al. Human HOXA5 homeodomain enhances protein transduction and its application to vascular inflammation. Biochem Biophys Res Commun. 2011;410(2):312-316.

65. Myers C, Charboneau A, Cheung I, Hanks D, Boudreau N. Sustained expression of homeobox D10 inhibits angiogenesis. Am J Pathol. 2002; 161(6):2099-2109.

66. Rhoads K, Arderiu G, Charboneau A, Hansen SL, Hoffman W, Boudreau N. A role for Hox A5 in regulating angiogenesis and vascular patterning. Lymphat Res Biol. 2005;3(4):240-252.

67. Arderiu G, Cuevas I, Chen A, Carrio M, East L,
Boudreau NJ. HoxA5 stabilizes adherens junctions via increased Akt1. Cell Adh Migr. 2007; 1(4):185-195.

68. Zhu Y, et al. Restoring transcription factor HoxA5 expression inhibits the growth of experimental hemangiomas in the brain. J Neuropathol Exp Neurol. 2009;68(6):626-632.

69. Chen $\mathrm{H}$, et al. Identification of transcriptional targets of HOXA5. J Biol Chem. 2005;280(19):19373-19380.

70. Miftakhova R, Sandberg T, Hedblom A, Nevzorova T, Persson JL, Bredberg A. DNA methylation in ATRA-treated leukemia cell lines lacking a PMLRAR chromosome translocation. Anticancer Res. 2012;32(11):4715-4722.

71. Iguchi-Ariga SM, Schaffner W. CPG methylation of the cAMP-responsive enhancer/promoter sequence TGACGTCA abolishes specific factor binding as well as transcriptional activation. Genes Dev. 1989; 3(5):612-619.

72. Zhang X, et al. Genome-wide analysis of cAMP-response element binding protein occupancy, phosphorylation, and target gene activation in human tissues. Proc Natl Acad Sci U S A. 2005; 102(12):4459-4464.

73. Boo YC. Shear stress stimulates phosphorylation of protein kinase A substrate proteins including endothelial nitric oxide synthase in endothelial cells. Exp Mol Med. 2006;38(1):63-71.

74. Schmittgen TD, Livak KJ. Analyzing real-time PCR data by the comparative C(T) method. Nat Protoc. 2008;3(6):1101-1108.

75. Newman M, Blyth BJ, Hussey DJ, Jardine D, Sykes PJ, Ormsby RJ. Sensitive quantitative analysis of murine LINE1 DNA methylation using high resolution melt analysis. Epigenetics. 2012;7(1):92-105.

76. Chambers JM, Hastie TJ, eds. Statistical Models in $S$. Pacific Grove, California, USA: Wadsworth and Brooks/Cole; 1992.

77. Wilkinson GN, Rogers CE. Symbolic descriptions of factorial models for analysis of variance. Applied Statistics. 1973;22(3):392-399.

78. Li LC, Dahiya R. MethPrimer: designing primers for methylation PCRs. Bioinformatics. 2002; 18(11):1427-1431. 